\title{
Non-Newtonian effects in the peristaltic flow of a Maxwell fluid
}

\author{
David Tsiklauri ${ }^{1}$ and Igor Beresnev ${ }^{2}$ \\ ${ }^{1}$ Space and Astrophysics Group, Physics Department, University of Warwick, Coventry, CV4 7AL, UK email: \\ tsikd@astro.warwick.ac.uk; ${ }^{2}$ Department of Geological and Atmospheric Sciences, Iowa State University, 253 Science I, Ames, \\ IA 50011-3212, U.S.A. email: beresnev@iastate.edu
}

\begin{abstract}
We analyzed the effect of viscoelasticity on the dynamics of fluids in porous media by studying the flow of a Maxwell fluid in a circular tube, in which the flow is induced by a wave traveling on the tube wall. The present study investigates novelties brought about into the classic peristaltic mechanism by inclusion of non-Newtonian effects that are important, for example, for hydrocarbons. This problem has numerous applications in various branches of science, including stimulation of fluid flow in porous media under the effect of elastic waves. We have found that in the extreme nonNewtonian regime there is a possibility of a fluid flow in the direction opposite to the propagation of the wave traveling on the tube wall.
\end{abstract}

47.55.Mh; 47.60.+i; 68.45.-v; 68.45.Kg; 92.10.Cg

\section{INTRODUCTION}

Investigation of flow dynamics of a fluid in a tube having circular cross-section, induced by a wave traveling on its wall (boundary), has many applications in various branches of science. The physical mechanism of the flow induced by the traveling wave can be well understood and is known as the so-called peristaltic transport mechanism. This mechanism is a natural cause of motion of fluids in the body of living creatures, and it frequently occurs in the organs such as ureters, intestines and arterioles. Peristaltic pumping is also used in medical instruments such as heart-lung machine etc.[1].

Laboratory experiments have shown that an external sonic radiation can considerably increase the flow rate of a liquid through a porous medium (Refs.[1,2] and references therein). Initially, the idea of flow stimulation via waves traveling on the flow boundary, in the context of porous media, has been proposed by Ganiev and collaborators [3]. They proposed that sonic radiation generates traveling waves on the pore walls in a porous medium. These waves, in turn, generate net flow of fluid via the peristaltic mechanism. Later, this problem has been studied in a number of publications, where authors used different simplifying assumptions in order to solve the problem (see e.g. Ref.[4]). The most recent and general study of stimulation of fluid flow in porous media via peristaltic mechanism is presented in Ref.[1], which we will use as a starting point in order to include non-Newtonian effects into the peristaltic model.

It is clear that a usual peristaltic mechanism discussed, e.g., in Ref.[1] can be used to describe the behavior of a classic Newtonian fluid; however, for example, oil and other hydrocarbons exhibit significant non-Newtonian behavior [5]. The aim of this paper is therefore to incorporate non-Newtonian effects into the classical peristaltic mechanism [1]. Thus, the present work formulates a realistic model of the peristaltic mechanism which is applicable to the non-Newtonian fluids (e.g. hydrocarbons) and not only to the Newtonian ones (e.g. ordinary water) which have been extensively investigated in the past [1].

It should be noted that there were similar studies in the past ([6] and references therein). However, the previous contributions discussed peristaltic mechanism for rheological equations other than the Maxwellian one. Thus, the present study fills this gap in the literature. In addition, this study is motivated by the recent results of del Rio, de Haro and Whitaker [7] and Tsiklauri and Beresnev [8], who found novel effects, including the enhancement of a Maxwellian fluid flow in a tube that is subjected to an oscillatory pressure gradient.

\section{THE MODEL}

We consider an axisymmetric cylindrical tube (pore) of radius $R$ and length $L$. We assume that elastic wave induces a traveling wave on the wall (boundary) of the tube with the displacement of the following form:

$$
W(z, t)=R+a \cos \left(\frac{2 \pi}{\lambda}(z-c t)\right)
$$

where $a$ is the amplitude of the traveling wave, while $\lambda$ and $c$ are its wave-length and velocity, respectively. We note that $z$-axis of the $(r, \phi, z)$ cylindrical coordinate system is directed along the axis of the tube. 
The equations which govern the flow are the balance of mass

$$
\frac{\partial \rho}{\partial t}+\nabla \cdot(\rho \vec{v})=0
$$

and the momentum equation

$$
\rho \frac{\partial \vec{v}}{\partial t}+\rho(\vec{v} \nabla) \vec{v}=-\nabla p-\nabla \tilde{\tau}
$$

where $\rho, p$ and $\vec{v}$ are the fluid density, pressure and velocity, respectively; $\tilde{\tau}$ represents the viscous stress tensor. We describe the viscoelastic properties of the fluid using the Maxwell's model [7], which assumes that

$$
t_{m} \frac{\partial \tilde{\tau}}{\partial t}=-\mu \nabla \vec{v}-\frac{\mu}{3} \nabla \cdot \vec{v}-\tilde{\tau}
$$

where $\mu$ is the viscosity coefficient and $t_{m}$ is the relaxation time.

We further assume that the following equation of state holds

$$
\frac{1}{\rho} \frac{d \rho}{d p}=\kappa
$$

where $\kappa$ is the compressibility of the fluid. We also assume that the fluid's velocity has only $r$ and $z$ components.

We make use of "no-slip" boundary condition at the boundary of the tube, i.e.

$$
v_{r}(W, z, t)=\frac{\partial W}{\partial t}, \quad v_{z}(W, z, t)=0 .
$$

Eq.(4) can be re-written in the following form:

$$
\left(1+t_{m} \frac{\partial}{\partial t}\right) \tilde{\tau}=-\mu \nabla \vec{v}-\frac{\mu}{3} \nabla \cdot \vec{v}
$$

Further, we apply the operator $\left(1+t_{m} \partial / \partial t\right)$ to the momentum equation (3) and eliminate $\tilde{\tau}$ in it using Eq.(7):

$$
-\left(1+t_{m} \frac{\partial}{\partial t}\right) \nabla p+\mu \nabla^{2} \vec{v}+\frac{\mu}{3} \nabla(\nabla \cdot \vec{v})=\left(1+t_{m} \frac{\partial}{\partial t}\right)\left[\rho \frac{\partial \vec{v}}{\partial t}+\rho(\vec{v} \nabla) \vec{v}\right] .
$$

The equations are made dimensionless by scaling the length by $R$ and time by $R / c$. Also, we have introduced the following dimensionless variables (and have omitted the tilde sign in the latter equations): $\tilde{W}=W / R, \tilde{\rho}=\rho / \rho_{0}$, $\tilde{v}_{r}=v_{r} / c, \tilde{v}_{z}=v_{z} / c, \tilde{p}=p /\left(\rho_{0} c^{2}\right)$. Here, $\rho_{0}$ is the regular (constant) density at the reference pressure $p_{0}$. We have also introduced $\epsilon=a / R, \alpha=2 \pi R / \lambda, R e=\rho_{0} c R / \mu, \chi=\kappa \rho_{0} c^{2}$.

Following Ref.[1], we seek the solution of the governing equations in a form:

$$
\begin{gathered}
p=p_{0}+\epsilon p_{1}(r, z, t)+\epsilon^{2} p_{2}(r, z, t)+\ldots, \\
v_{r}=\epsilon u_{1}(r, z, t)+\epsilon^{2} u_{2}(r, z, t)+\ldots \\
v_{z}=\epsilon v_{1}(r, z, t)+\epsilon^{2} v_{2}(r, z, t)+\ldots \\
\rho=1+\epsilon \rho_{1}(r, z, t)+\epsilon^{2} \rho_{2}(r, z, t)+\ldots
\end{gathered}
$$

Then, doing a usual perturbative analysis using the latter expansions, we can obtain a closed set of governing equations for the first $(\epsilon)$ and second $\left(\epsilon^{2}\right)$ order.

Further, following the authors of $[1,8]$, we seek the solution of the liner problem in the form:

$$
\begin{aligned}
& u_{1}(r, z, t)=U_{1}(r) e^{i \alpha(z-t)}+\bar{U}_{1}(r) e^{-i \alpha(z-t)}, \\
& v_{1}(r, z, t)=V_{1}(r) e^{i \alpha(z-t)}+\bar{V}_{1}(r) e^{-i \alpha(z-t)},
\end{aligned}
$$




$$
\begin{gathered}
p_{1}(r, z, t)=P_{1}(r) e^{i \alpha(z-t)}+\bar{P}_{1}(r) e^{-i \alpha(z-t)}, \\
\rho_{1}(r, z, t)=\chi P_{1}(r) e^{i \alpha(z-t)}+\chi \bar{P}_{1}(r) e^{-i \alpha(z-t)} .
\end{gathered}
$$

Here and in the following equations the bar denotes a complex conjugate.

On the other hand, we seek the second $\left(\epsilon^{2}\right)$ order solution in the form:

$$
\begin{aligned}
& u_{2}(r, z, t)=U_{20}(r)+U_{2}(r) e^{i 2 \alpha(z-t)}+\bar{U}_{2}(r) e^{-i 2 \alpha(z-t)}, \\
& v_{2}(r, z, t)=V_{20}(r)+V_{2}(r) e^{i 2 \alpha(z-t)}+\bar{V}_{2}(r) e^{-i 2 \alpha(z-t)}, \\
& p_{2}(r, z, t)=P_{20}(r)+P_{2}(r) e^{i 2 \alpha(z-t)}+\bar{P}_{2}(r) e^{-i 2 \alpha(z-t)}, \\
& \rho_{2}(r, z, t)=D_{20}(r)+D_{2}(r) e^{i 2 \alpha(z-t)}+\bar{D}_{2}(r) e^{-i 2 \alpha(z-t)} .
\end{aligned}
$$

The latter choice of solution is motivated by the fact that the peristaltic flow is essentially a non-linear (second order) effect [1], and adding a non-oscillatory term in the first order gives only trivial solution. Thus, we can add non-oscillatory terms, such as $U_{20}(r), V_{20}(r), P_{20}(r), D_{20}(r)$, which do not cancel out in the solution after the time averaging over the period, only in the second and higher orders.

In the first order by $\epsilon$ we obtain:

$$
\begin{gathered}
-\left(1-i \alpha t_{m}\right) P_{1}^{\prime}+\frac{1}{R e}\left(U_{1}^{\prime \prime}+\frac{U_{1}^{\prime}}{r}-\frac{U_{1}}{r^{2}}-\alpha^{2} U_{1}\right)+\frac{1}{3 R e} \frac{d}{d r}\left(U_{1}^{\prime}+\frac{U_{1}}{r}+i \alpha V_{1}\right)=-i \alpha\left(1-i \alpha t_{m}\right) U_{1}, \\
-i \alpha\left(1-i \alpha t_{m}\right) P_{1}^{\prime}+\frac{1}{R e}\left(V_{1}^{\prime \prime}+\frac{V_{1}^{\prime}}{r}-\alpha^{2} V_{1}\right)+\frac{i \alpha}{3 R e}\left(U_{1}^{\prime}+\frac{U_{1}}{r}+i \alpha V_{1}\right)=-i \alpha\left(1-i \alpha t_{m}\right) V_{1}, \\
\left(U_{1}^{\prime}+\frac{U_{1}}{r}+i \alpha V_{1}\right)=i \alpha \chi P_{1} .
\end{gathered}
$$

Here, the prime denotes a derivative with respect to $r$.

Further, we re-write the system (9)-(11) in the following form:

$$
\begin{gathered}
-\gamma P_{1}^{\prime}+\left(U_{1}^{\prime \prime}+\frac{U_{1}^{\prime}}{r}-\frac{U_{1}}{r^{2}}-\beta^{2} U_{1}\right)=0, \\
-\gamma P_{1}-\frac{i}{\alpha}\left(V_{1}^{\prime \prime}+\frac{V_{1}^{\prime}}{r}-\beta^{2} V_{1}\right)=0
\end{gathered}
$$

where

$$
\gamma=\left(1-i \alpha t_{m}\right) R e-i \alpha \chi / 3, \quad \beta^{2}=\alpha^{2}-i \alpha\left(1-i \alpha t_{m}\right) R e .
$$

Note, that Eqs.(12)-(13) are similar to Eq.(3.11) from Ref.[1], except that $\gamma$ and $\beta$ are modified by substitution $R e \rightarrow\left(1-i \alpha t_{m}\right) R e$.

Repeating the analysis similar to the one from Ref.[1], we obtain the master equation for $U_{1}(r)$ and find its general solution as

$$
U_{1}(r)=C_{1} I_{1}(\nu r)+C_{2} I_{1}(\beta r),
$$

where $I_{1}$ is the modified Bessel function of the first kind of order 1 ; and $C_{1}$ and $C_{2}$ are complex constants defined by

$$
C_{1}=\frac{\alpha \beta \nu i I_{0}(\beta)}{2\left[\alpha^{2} I_{0}(\nu) I_{1}(\beta)-\beta \nu I_{0}(\beta) I_{1}(\nu)\right]}, \quad C_{2}=\frac{-\alpha^{3} i I_{0}(\nu)}{2\left[\alpha^{2} I_{0}(\nu) I_{1}(\beta)-\beta \nu I_{0}(\beta) I_{1}(\nu)\right]},
$$


where

$$
\nu^{2}=\alpha^{2} \frac{(1-\chi)\left(1-i \alpha t_{m}\right) R e-(4 / 3) i \alpha \chi}{\left(1-i \alpha t_{m}\right) R e-(4 / 3) i \alpha \chi} .
$$

Here, $I_{0}$ is the modified Bessel function of the first kind of order 0.

We also obtain the general solution for $V_{1}(r)$ :

$$
V_{1}(r)=\frac{i \alpha C_{1}}{\nu} I_{0}(\nu r)+\frac{i \beta C_{2}}{\alpha} I_{0}(\beta r) .
$$

The second-order solution $V_{20}(r)$ can also be found in a way similar to the one used in Ref.[1]:

$$
V_{20}(r)=D_{2}-\left(1-i \alpha t_{m}\right) \operatorname{Re} \int_{r}^{1}\left[V_{1}(\zeta) \bar{U}_{1}(\zeta)+\bar{V}_{1}(\zeta) U_{1}(\zeta)\right] d \zeta
$$

where $D_{2}$ is a constant defined by

$$
D_{2}=-\frac{i \alpha C_{1}}{2} I_{1}(\nu)-\frac{i \beta^{2} C_{2}}{2 \alpha} I_{1}(\beta)+\frac{-i \alpha \bar{C}_{1}}{2} I_{1}(\bar{\nu})+\frac{i\left(\beta^{2} C_{2}\right)}{2 \alpha} I_{1}(\bar{\beta}) .
$$

The net dimensionless fluid flow rate $Q$ can be calculated as [1]:

$$
Q(z, t)=2 \pi\left[\epsilon \int_{0}^{1} v_{1}(r, z, t) r d r+\epsilon^{2} \int_{0}^{1} v_{2}(r, z, t) r d r+O\left(\epsilon^{3}\right)\right] .
$$

In order to obtain the net flow rate averaged over one period of time, we have to calculate

$$
<Q>=\frac{\alpha}{2 \pi} \int_{0}^{2 \pi / \alpha} Q(z, t) d t .
$$

This time averaging yields

$$
<Q>=2 \pi \epsilon^{2} \int_{0}^{1} V_{20}(r) r d r
$$

or finally substituting the explicit form of $V_{20}(r)$ we obtain for the dimensionless net flow rate

$$
<Q>=\pi \epsilon^{2}\left[D_{2}-\left(1-i \alpha t_{m}\right) R e \int_{0}^{1} r^{2}\left[V_{1}(r) \bar{U}_{1}(r)+\bar{V}_{1}(r) U_{1}(r)\right] d r\right]
$$

\section{NUMERICAL RESULTS}

In the previous section, we have shown that the inclusion of non-Newtonian effects into the classical peristaltic mechanism by using the Maxwell fluid model yields the following change: $R e \rightarrow\left(1-i \alpha t_{m}\right) R e$ in all of the solutions.

It is known that the viscoelastic fluids described by the Maxwell model have different flow regimes depending on the value of the parameter $D e=t_{v} / t_{m}$, which is called the Deborah number [7]. In effect, Deborah number is a ratio of the characteristic time of viscous effects $t_{v}=\rho R^{2} / \mu$ to the relaxation time $t_{m}$. As noted in Ref. [7], the value of the parameter $D e$ (which the authors of Ref.[7] actually call $\alpha$ ) determines in which regime the system resides. Beyond a certain critical value $\left(D e_{c}=11.64\right)$, the system is dissipative, and conventional viscous effects dominate. On the other hand, for small $D e\left(D e<D e_{c}\right)$ the system exhibits viscoelastic behavior.

A numerical code has been written to calculate $\langle Q\rangle$ according to Eq.(16). In order to check the validity of our code, we run it for the parameters similar to the ones used by other authors. For instance, for $\epsilon=0.15, R e=100.00$, $\alpha=0.20, \chi=0, t_{m}=0$ we obtain $\langle Q\rangle=0.2708706458$, which is equal (if we keep 4 digits after the decimal point) to the result of the authors of Ref.[1] who actually obtained $\langle Q\rangle=0.2709$.

Further, we have made several runs of our code for different values of the parameter $t_{m}$. We note again that $t_{m}$ enters the equations because we have included non-Newtonian effects into our model. Eq.(16) will be identical to the similar Eq.(4.1) from Ref.[1] if we set $t_{m}=0$ in all our equations. 
The results of our calculation are presented in Fig.1, where we investigate the dependence of $<Q>$ on the compressibility parameter $\chi$ for the various values of $t_{m}$. In order to compare our results with the ones from Ref.[1], we have plotted $\langle Q\rangle$ for the following set of parameters: $\epsilon=0.001, R e=10000.00, \alpha=0.001, t_{m}=0$ (solid line). We note that the curve is identical to the corresponding curve in Fig.2 from Ref.[1]. This obviously corroborates the validity of our numerical code. Further, to investigate the dependence of the flow rate $\langle Q\rangle$ on $t_{m}$, we perform the calculation for a few values of $t_{m}$. When $t_{m}=1.0$, we notice no noticeable change in the plot as both curves coincide within the plotting accuracy. For $t_{m}=100.00$ (dashed curve with crosses), we notice slight deviation from the Newtonian limiting case (solid line), which translates into shifting the maximum towards larger $\chi$ 's. For $t_{m}=1000.00$ (dash-dotted curve with asterisks), we notice further deviation from the Newtonian flow, which also translates into shifting the maximum towards larger $\chi$ 's. However, for $t_{m}=10000.00$ (dashed curve with empty squares), we note much more drastic changes, including the absence of a maximum and rapid growth of $\langle Q\rangle$ in the considered interval of variation of compressibility parameter $\chi$. The observed pattern conforms to our expectation, since large $t_{m}$ means small $D e\left(D e<D e_{c}\right)$ and the system exhibits strong viscoelastic behavior. Note that $t_{m}$ is dimensionless and scaled by $R / c$.

After the above discussion it is relevant to define quantitatively the transition point where the flow starts to exhibit (non-Newtonian) viscoelastic effects. It is known [7] that $D e=t_{v} / t_{m}=\left(\rho R^{2}\right) /\left(\mu t_{m}\right)$. Now, using definition of $R e=\rho c R / \mu$ we can define critical value of $t_{m}$ as

$$
t_{m C}=\left(\frac{R e}{D e_{c}}\right) \frac{R}{c} .
$$

In all our figures we have used $R e=10000.0$. If we put the latter value of $R e$ and the critical value of the Deborah number 11.64 [7] into Eq.(17) we obtain $t_{m C}=859.11$ (measured in units of $R / c$ ). Therefore, the values of $t_{m}$ greater than $t_{m C}$ (for a given $R e$ ) correspond to sub-critical $\left(D e<D e_{c}\right)$ Deborah numbers for which viscoelastic effects are pronounced.

In Fig.2 we investigate the behavior of the flow rate $\langle Q\rangle$ on the parameter $\alpha$, which is the tube radius measured in wave-lengths. Again, to check for the consistency of our numerical results with the ones from Ref.[1] and also investigate novelties brought about by the introduction of non-Newtonian effects (appearance of non-zero relaxation time $t_{m}$ ) into the model, we first plot $\langle Q\rangle$ versus $\alpha$ for the following set of parameters: $\epsilon=0.001, R e=10000.00$, $\chi=0.6, t_{m}=0$. If we compare the solid curve in our Fig.2 with the dashed curve in Fig.3 of Ref.[1], we will note no difference, which again corroborates the validity of our numerical code. We then set $t_{m}$ to various non-zero values and investigate the changes introduced by non-Newtonian effects. As in Fig.1, we notice no change for $t_{m}=1.0$. For $t_{m}=100.00$ and $t_{m}=1000.00$, we notice that flow rate somewhat changes attaining lower values as $\alpha$ (radius of the tube) increases.

We treat the latter, $t_{m}=1000.00$, case separately for the reason of an appearance of a novel effect of negative flow rates when the interval of variation of $\alpha$ is increased up to 0.05. Again, we expect that for large $t_{m}\left(t_{m}>t_{m C}=\right.$ 859.11), i.e. small $D e\left(D e<D e_{c}\right)$, the system should exhibit viscoelastic behavior. We note from Fig. 3 that for $\alpha \geq 0.035,<Q>$ becomes negative, i.e., we observe backflow. In fact, by doing parametric study we conclude that $t_{m C}$ is the critical value of $t_{m}$ above which we observe backflow. By increasing $t_{m}$ further, $t_{m}=10000.00$, we note from Fig.4 that in this deeply non-Newtonian regime $\langle Q\rangle$ becomes highly oscillatory, but what is unusual again is that we observe the negative flow rates for certain values of $\alpha$ that is the tube radius measured in wave-lengths. Obviously, the negative $\langle Q>$ means that flow occurs in the direction opposite to the direction of propagation of traveling wave on the tube wall. Oscillatory behavior (appearance of numerous of maxima in the behavior of a physical variable) in the deeply non-Newtonian regime is not new [8]. However, the flow of a fluid created by the peristaltic mechanism in the direction opposite to the direction of propagation of traveling wave is unusual and should be attributed to a complicated, non-linear form of the response of a Maxwell fluid to the stress exerted by the wave.

\section{CONCLUSIONS}

In this paper, we investigated the dynamics of fluid flow in a tube with a circular cross-section, induced by a wave traveling on its wall (boundary). This problem has numerous applications in various branches of science, including stimulation of fluid flow in porous media under the effect of elastic waves.

The present study investigates novelties brought about into the classic peristaltic mechanism by the inclusion of non-Newtonian effects based on the model of Maxwell fluid.

We have found that in the extreme non-Newtonian regime there is a possibility of flow in the direction opposite to the propagation of the wave traveling on the tube wall. Somewhat similar effect is known as the acoustic streaming [9], in which an acoustic wave propagating in a tube induces a mean flow in the direction of propagation in the acoustic 
boundary layer, but in the opposite direction in the central part of the tube. The mean flow or acoustic streaming is caused by the presence of friction at the bounding surfaces of the tube. While fluid away from the neighborhood of a boundary vibrates irrotationally as the acoustic wave passes, fluid in close proximity to the boundary must vibrate rotationally to satisfy the no-slip condition on the tube wall. This deviation from inviscid, irrotational behavior provides an effective driving force known as the Reynolds stress. This effective force, because of it is quadratic rather than linear, has non-vanishing time-average tangential component to the tube wall that drives flow in the boundary layer. In the case considered in our paper instead of having acoustic wave propagating through the volume of the tube, we have a wave traveling of the tube wall, besides we have further complication of considering non-Newtonian (Maxwell) fluid (recall that the discovered effect is demonstrated for the case of non-Newtonian regime $t_{m} / t_{m C}>1.0$ ). Similarly, the peristaltic flow itself is a second order non-linear effect. Therefore, the flow of a fluid created by the peristaltic mechanism in the direction opposite to the direction of propagation of traveling wave (i.e. back-flow) could be explained by a complicated, non-Newtonian, non-linear response of a Maxwell fluid to the stress exerted by the traveling wave.

\section{ACKNOWLEDGMENTS}

This work was supported by the Iowa State University Center for Advanced Technology Development and ETREMA Products, Inc. We would like to thank anonymous referee for useful suggestions of improvement of the manuscript.

[1] A.C.T. Aarts, G. Ooms, J. Eng. Math., 34, 435 (1998)

[2] I.A. Beresnev, P.A. Johnson, Geophys., 59, 1000 (1994); T. Drake, I. Beresnev, The American Oil \& Gas Reporter, September 1999, p.101

[3] R.F. Ganiev, L.E. Ukrainskii, K.V. Frolov, Sov. Phys. Dolk., 34, 519 (1989)

[4] A.H. Shapiro, M.Y. Jaffrin, S.L. Weinberg, J.Fluid Mech., 37, 799 (1969); F. Yin and Y.C. Fung, J. Appl. Mech., 36, 579 (1969); S. Takabatake, K. Ayukawa, A. Mori, J. Fliud Mech., 193, 267 (1988)

[5] C. Chang, Q.D. Nguyen, H.P. Ronningsen, J. Non-Newtonian Fluid Mech., 87, 127 (1999); B.P. Williamson, K. Walters, T.W. Bates, R.C. Coy and A.L. Milton, J. Non-Newtonian Fluid Mech., 73, 115 (1997); G.A. Nunez, G.S. Ribeiro, M.S. Arney, J. Feng and D.D. Joseph, J. Rheol., 38(5), 1251 (1994); L.T. Wardhaugh and D.V. Boger, J. Rheol., 35(6), 1121 (1991)

[6] A.M. Siddiqui and W.H. Schwarz, J. Non-Newton. Fluid Mech., 53, 257 (1994)

[7] J.A. del Rio, M.L. de Haro and S. Whitaker, Phys. Rev., E58, 6323 (1998)

[8] D. Tsiklauri and I. Beresnev, Phys. Rev. E63, 046304 (2001)

[9] Q. Qi, R.E. Johnson and J.G. Harris, J. Acoust. Soc. Am., 97, 1499 (1995)

\section{Figure captions}

Fig. 1 Dimensionless flow rate $\langle Q\rangle$ as a function of compressibility parameter $\chi$. The parameters used are $\epsilon=0.001, R e=10000.00, \alpha=0.001 . t_{m}=0$ corresponds to the solid line, whereas $t_{m}=100.00,1000.00$ and 10000.00 correspond to the dashed curve with crosses, dash-dotted curve with asterisks, and dashed curve with empty squares, respectively.

Fig. 2 Plot of dimensionless flow rate $\langle Q\rangle$ as a function of $\alpha$. Here, $\epsilon=0.001, R e=10000.00, \chi=0.6 . t_{m}=0$ corresponds to the solid line, whereas $t_{m}=100.00$ and 1000.00 correspond to the dashed curve with crosses and dash-dotted curve with asterisks, respectively.

Fig. 3 Plot of dimensionless flow rate $\langle Q\rangle$ as a function of $\alpha$ on a larger than in Fig.2 interval of variation of $\alpha$. Here, $\epsilon=0.001, R e=10000.00, \chi=0.6, t_{m}=1000.00$.

Fig. 4 Plot of dimensionless flow rate $\langle Q\rangle$ as a function of $\alpha$. Here, $\epsilon=0.001, R e=10000.00, \chi=0.6$, $t_{m}=10000.00$. 


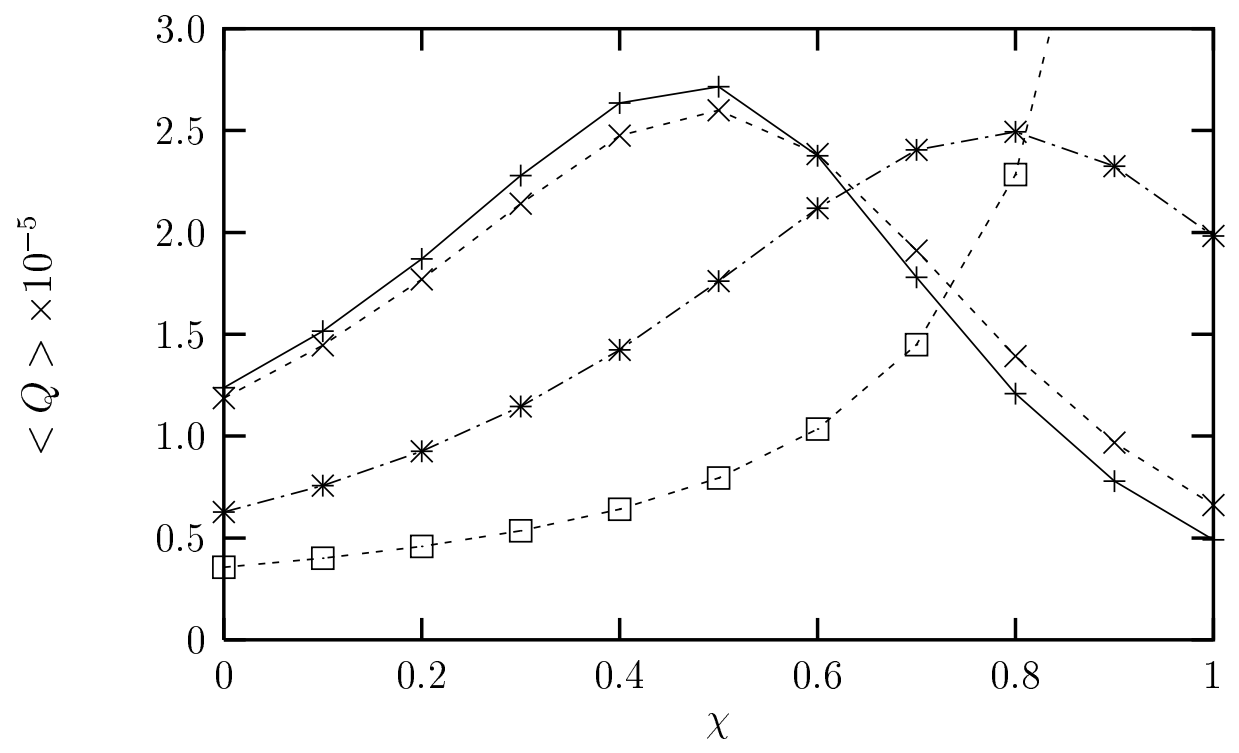




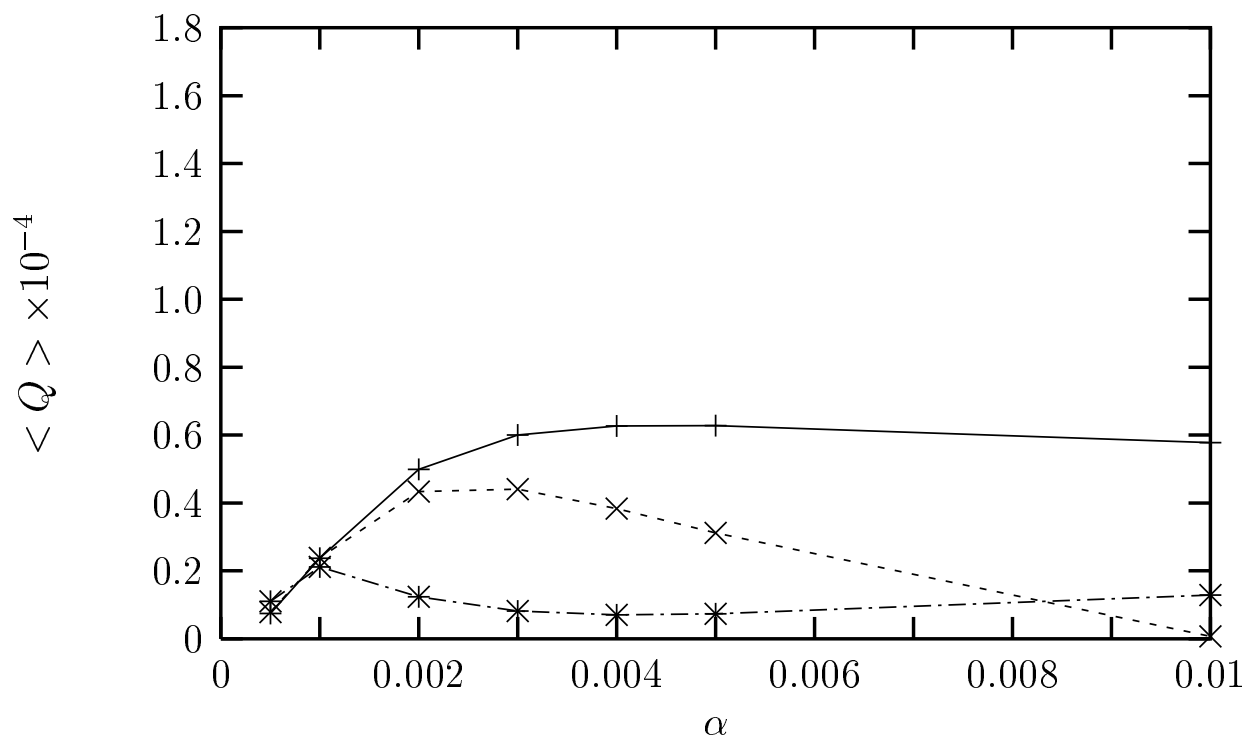




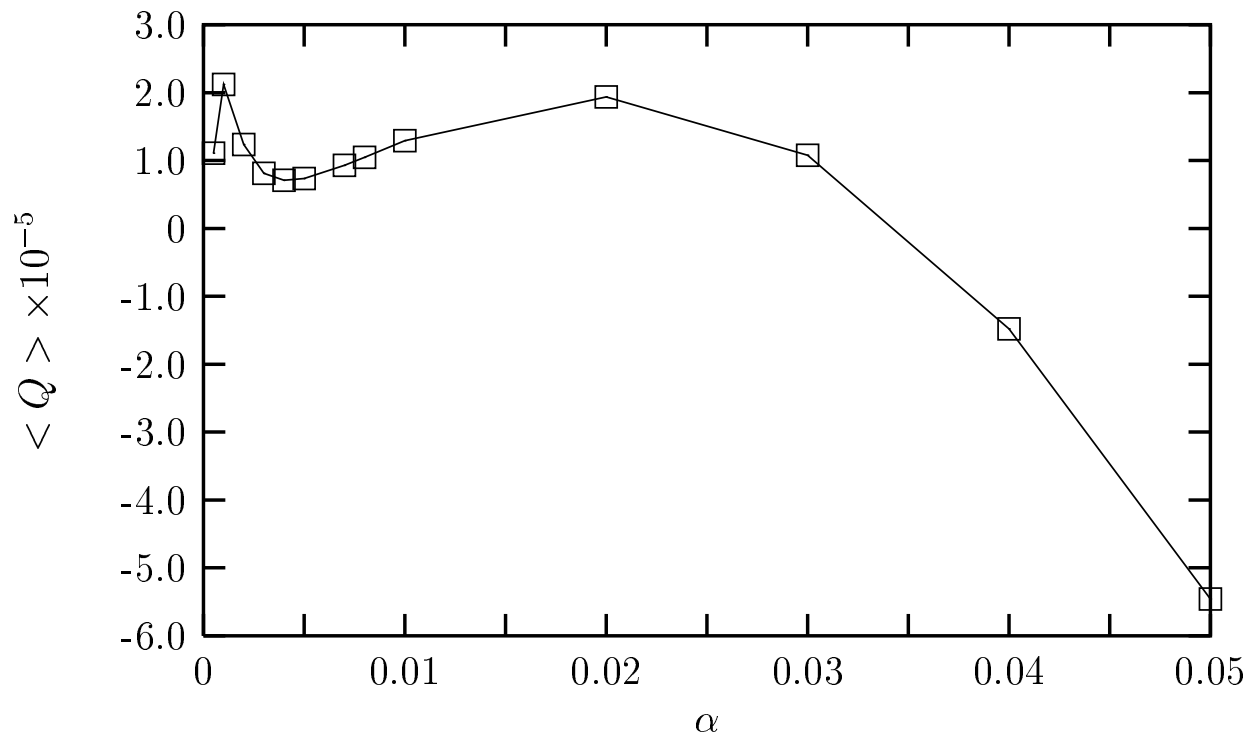




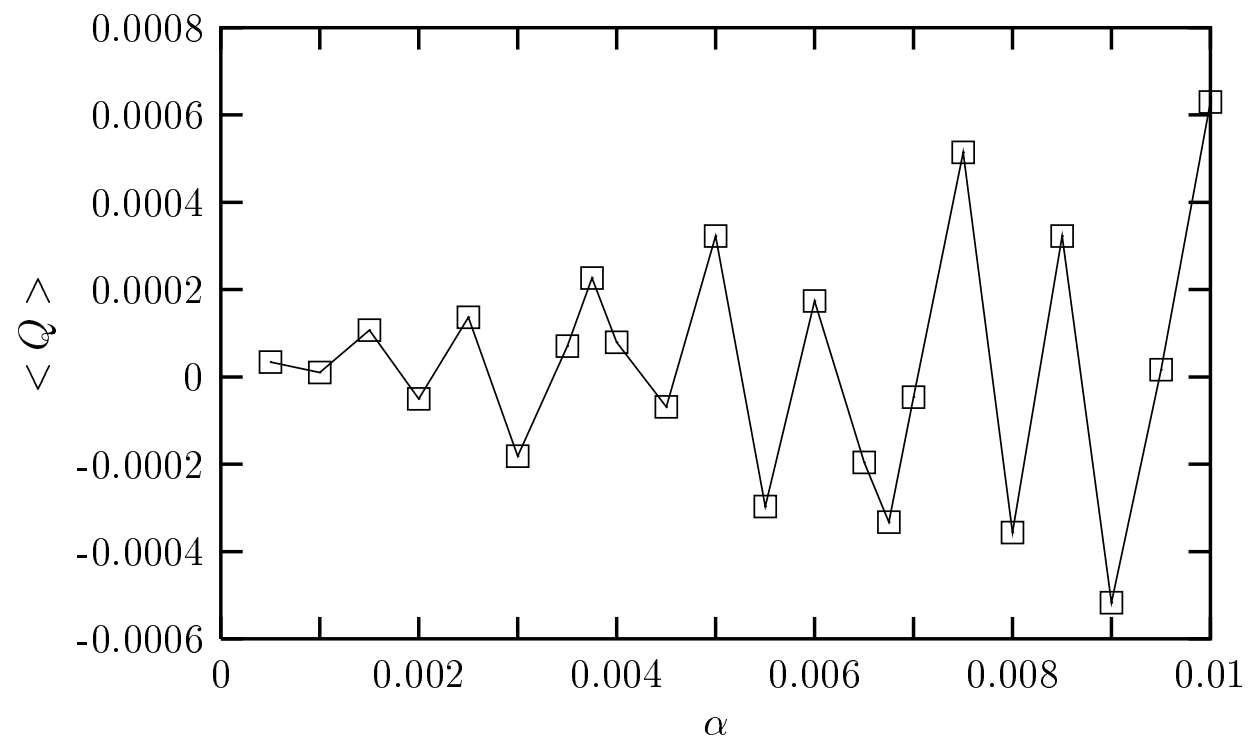

